\title{
CALIBRATION OF AN AGENT-BASED SIMULATION MODEL DEPICTING A REFUGEE CAMP SCENARIO
}

\author{
Rachel T. Johnson \\ Operations Research Department \\ Naval Postgraduate School \\ 1411 Cunningham Rd. \\ Monterey, CA 93943, USA
}

\author{
Thorsten A. Lampe \\ System Design Centre Germany \\ European Aeronautic Defence and \\ Space Company (EADS) \\ 85716 Unterschleissheim, Germany
}

\author{
Stephan Seichter \\ Modeling and Simulation Department \\ Bundeswehr Transformation Center \\ 85521 Ottobrunn, Germany
}

\begin{abstract}
This paper describes the use of the design and analysis of experiments to calibrate an agent-based simulation model of a peace-keeping mission. The situation depicted is a refugee camp scenario in which de-escalation of enraged civilians is required by military personnel. Calibration was determined by ensuring that the military actions in the simulation properly followed the typical rules of engagement and the expected reactions of both civilians and military forces. The expectations were determined by subject matter experts including active military personnel and psychologists.
\end{abstract}

\section{INTRODUCTION}

Understanding the complex dynamics of human behavior and interaction is extremely important for peace support missions. Recent war activity and relocation of civilians to refugee camps have given way to a new set of challenges for military forces. The challenges faced by military personnel stationed in a refugee camp are quite different from those in a classic conflict situation such as a war zone. For example, military forces stationed in a refugee camp are usually not confronted with a heavily armed enemy, but with hungry, scared, or in some cases enraged civilians. The involvement of military forces requires the understanding of the given situation and contextual behaviors of the people involved in the situation. In the case of the refugee scenario, it is necessary to understand how civilians will react within the camps. Important questions to understand include: Will the civilians in the refugee camp remain peaceful or will they become aggressive? Should the soldiers keep a low profile or take strong actions to maintain peace in the camp? What level of involvement is necessary to de-escalate a situation created by an enraged civilian? Are the current rules of engagement adequate or should they be modified to better address issues that may arise in a refugee camp? The answers to these questions are required for the operational and tactical aspects of military involvement in a refugee camp.

One viable option to address these concerns is through the application of simulation. In particular, the most challenging task of simulating human behavior is addressed by agent-based models. An agent-based simulation models interactions among individuals, making it well-suited for modeling sociological and psychological behavior and interactions of humans. Sanchez and Lucas (2002) describe agent-based simulations as models which are composed of agents: objects or entities that behave autonomously. In agent-based models the collective behavior of the simulated system is the result of the interactions and behaviors of the individually simulated agents.

PAX (the Latin word for peace) is an agent-based simulation model developed in Germany for the German Armed Forces and intended to aid in peace support operation (PSO) missions. PAX models peace-keeping scenarios with a focus on the behavior and interaction of both military and civilian personnel. The behavior patterns and interactions are based on empirical knowledge about the evolvement of aggression and de-escalation of the aggressors (see Mosler et al. 2005). The calibration of the PAX model developed to mimic civilian and military interaction and behavior in a refugee camp is described in this paper. Once the model is calibrated and validated, it will be used to determine adequate use of material and personnel in crisis regions and to study and potentially modify current rules of engagement (RoE).

Calibration is a crucial step in simulation modeling. This paper describes the calibration of a small and isolated situation in a refugee camp scenario. The calibrated model will ensure that the actions of military forces in the simulation model match the RoE, and that the reactions of civilians and military match the outcomes expected by subject matter experts such as active military personnel and psychologists. Section 2 describes the refugee camp model under study and the situation that is used for the calibration. Section 3 details the calibration method used in the paper. Specifically the set-up of the problem, identifi- 
cation of the input variables and output responses, and the design and analysis of experiments are discussed. This is followed by Section 4, which presents the results found in the study. Finally, the conclusions and discussion are given in Section 5.

\section{REFUGEE CAMP MODEL}

This paper describes the calibration of two simulated situations that take place in a refugee camp. Before covering the situations, or "vignettes," in more detail, a general overview of the PSO model PAX as well as the general scenario will be given.

\subsection{PSO Model PAX}

The German Armed Forces (Bundeswehr) have increasingly taken part in PSOs. A significant challenge is the development of adequate tactics, techniques and procedures (TTP) in these dynamic and hard-to-predict operations. However, due to their specific focus on providing help and de-escalating critical situations, peace support operations, including humanitarian assistance operations, can not be adequately modeled with existing combat models.

For these reasons the high-resolution, agent-based simulation model PAX was developed by the European Aeronautic Defence and Space Company (EADS) on behalf of the German Bundeswehr. PAX concentrates on modeling peacekeeping aspects and incorporates a complex, empirically based psychological theory on collective aggression including human factors such as motivation, emotions or stress. Besides military actions, psychological factors and their influence on the decisions and the behavior of all persons concerned may have a considerable effect on the development of an operation. The military forces modeled in PAX have the possibility to not only use different types of weapons, but to also take measures of active deescalation, such as trying to calm down people or talking to civilian leaders. The model incorporates a detailed representation of the individual actors and their internal states. The represented human behavior includes the modeling of emotions, such as fear or anger, which are considered crucial factors in the evolution of aggressive behavior, which military forces are frequently confronted with. In order to draw meaningful conclusions from the PAX simulation, the model must be calibrated and validated. A methodology for calibrating PAX in a given scenario context using experimental design and an iterative analysis is described in this paper.

\subsection{Refugee Camp Scenario}

The scenario to be calibrated is set in a refugee camp built and operated by Military Peace Keeping Forces. Each refugee is assigned a place in a specified tent, where each tent houses 10 refugees. The civilians in the camp all belong to the same ethnic group, but two different sub-groups (e.g. family clans or similar structures) are assumed.

It is further assumed that the threat level around the camp is calm, so that external disturbances are not expected. Within the camp, the force provider's law and typical RoE are applied with regard to the execution of force and how to react on criminal offenses. German law and RoE for PSO partly are applied in order to create a realistic scenario.

The distribution of meals is organized by the Military. Food is distributed to one representative per tent in the form of ready-to-eat meals that the representative collects. The collections occur at specified and well-known distribution times in a dedicated food distribution area. This area is separated from the rest of the camp by a barbwire fence. Figure 1 gives a bird's eye view of the refugee camp scenario.



Figure 1: Refugee Camp Scenario 
Within this refugee camp scenario, the modeled vignettes cover a situation where a member of one group (B) of the camp has already received food packages and is on his way back to his tent. Members of another group (A) do not have their rations yet and attempt to get these packages from B. To isolate the relevant model effects of the relevant parameters, small vignettes with only 4 agents from the different groups will be examined.

Underlying questions for investigation that the model will be used to answer after the model is calibrated are:

- Are the applied RoE effective for the military and the given mission of operating a refugee camp?

- Can a secure environment for both, refugees and camp operators, e.g. military and an NGO, be established by applying these RoE?

These are the driving questions behind the activities in adjusting and calibrating PAX for later decision support with regard to assessing and comparing different TTPs and RoEs.

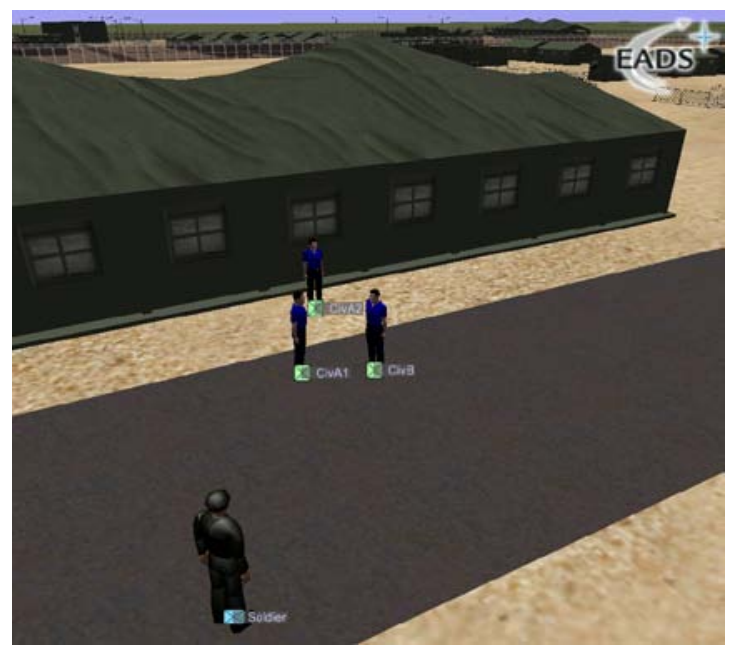

Figure 2: Detailed vignette within the refugee camp scenario

\section{CALIBRATION METHOD}

In order to use the simulation model to make decisions about effective military actions and evaluate current and potential RoE, it is important to use a calibrated simulation model. The calibration of a model that simulates human behavior and interaction is particularly challenging. The most difficult challenge is that there is no data source against which the model can be calibrated. Banks et al. (2001) describe the calibration of a simulation as an "iterative process of comparing the model to the real system, making adjustments (or even major changes) to the model, comparing the revised model to reality, making additional adjustments, comparing again, and so on." In the case of the refugee camp scenario described in this paper, there is not necessarily a "real system" to compare the simulation with. Because of this, it is necessary to calibrate the model against subject matter expert opinion of what will and should happen in a particular situation involving human interaction. For this particular reason, it is more desirable to perform the calibration on a very small scale situation. Thus for the work described in this paper a scenario was chosen in which a conflict takes place within the refugee camp among a small number of civilians. When the conflict takes place, a single military solider is present to de-escalate the situation. Such a situation may or may not happen in reality, but must be calibrated in order to ensure fidelity of the entire model.

Two scenarios were chosen as the basis for calibration of the refugee camp model. The first is referred to as the lowescalation scenario and the second is the high-escalation scenario. In the low-escalation scenario a civilian A from Group A tries to steal a food package from a civilian B from Group B who is walking from the food distribution center back to his tent within the camp. Civilian A verbally threatens civilian B in order to obtain the food package. In this scenario, a soldier witnesses the threats and tries to stop the food package from being stolen and prevent escalation of the situation by following the rules of engagement. The high-escalation scenario is exactly the same situation as the low-escalation scenario, but civilian A attacks civilian B with a knife in order to obtain the food as opposed to a verbal threat.

The calibration of these two scenarios was done using an iterative method described in the phases below: 
Phase 1: Pre-experimental phase

- Identify and classify input/output variables

- Select appropriate ranges and or levels of input variables

- Define measures of effectiveness (MOE)

- Select the experimental design

Phase 2: Run and analyze experiments to develop understanding and to exclude unrealistic settings

- Run the simulations prescribed by the experimental design

- Analyze the experiments graphically and statistically

Phase 3: Calibration

- Determine if the results from step 2 achieve the required MOEs - Is the model calibrated?

- If the model is calibrated, stop; else go back to Step 1

\section{RESULTS}

In this section, the details of the calibration steps are presented along with the results. This section is broken into sections corresponding to the steps presented in the calibration method (Section 3).

\subsection{Pre-Experimental Phase}

The most important step of this pre-experimental phase was to define the MOEs to calibrate against. Each specific vignette of the refugee camp scenario can then be declared as calibrated when the MOEs are met. Due to the lack of real system data the MOEs were instead determined by subject matter experts' (SME) expectations. The MOEs are shown in Table 1.

Table 1: MOEs and respective calibration goals

\begin{tabular}{|l|l|}
\hline MOE & Calibration Goal \\
\hline Low-escalation vignette & $>90 \%$ \\
\hline Soldier's success in preventing stealing & $0 \%$ \\
\hline Use of weapons by CivA & $0 \%$ \\
\hline Use of weapons by Soldier & $\geq 90 \%$ \\
\hline High-escalation vignette & $\geq 90 \%$ \\
\hline Soldier's success in preventing stealing & \\
\hline Soldier's success in preventing subsequent attacks & \\
\hline
\end{tabular}

Table 1 indicates that the SME expectation in the low-escalation vignette was that the soldier would successfully prevent the packages being stolen in more than $90 \%$ of the cases. Note that each simulation run results in a binary response - success (the package was not stolen) or failure (the package was stolen). We converted this binary response into a percentage by running each particular experimental scenario 100 times and calculating the MOE percentage as the number of successes divided by 100 .

To achieve the goal of creating a simulation that prevents the threatening civilian from stealing the package in $90 \%$ of the experimental trials, an experimental design technique was used that prescribed a sequence of experimental trials. These trials are created in a way that vary input factors simultaneously and allow their individual impact on the responses to be determined. Thus, the initial challenge in the calibration efforts was to identify the specific input factors of interest and their corresponding ranges.

Figure 3 shows an architectural high-level view of the PAX model with its inputs and outputs based on the PECS architecture which is described in Urban (2005) and was adapted in the Military PECS (MPECS) reference model for the specific purposes required in PAX. This figure hints at the large number of parameters and the complexity of the internal model. 


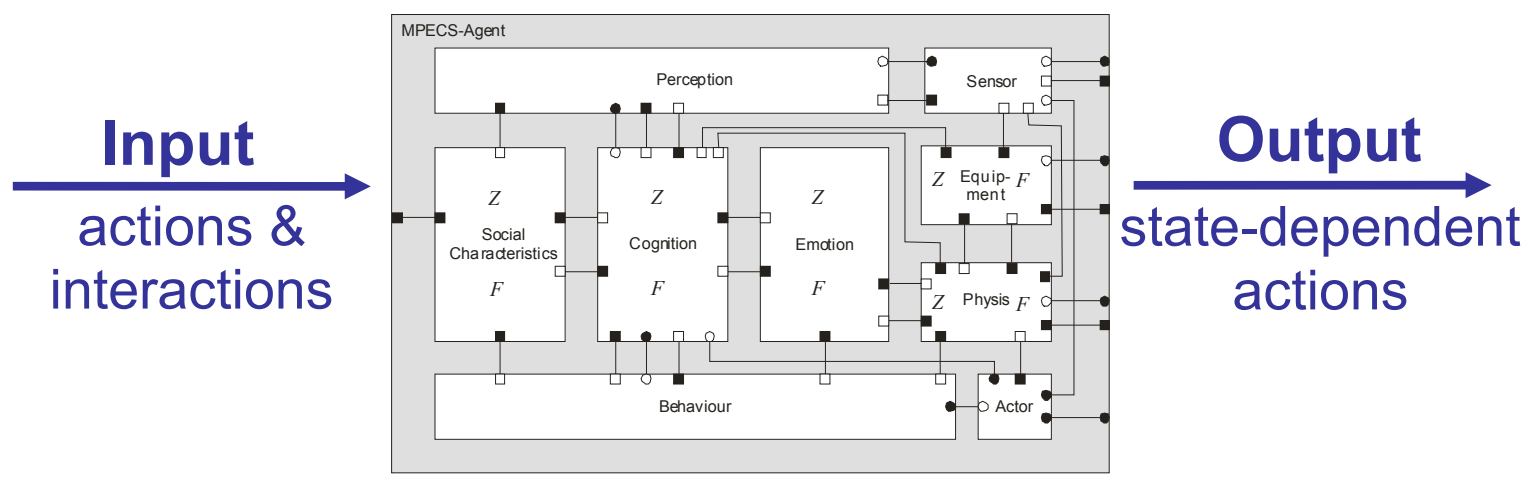

Figure 3: High-level view of PAX

The inputs of interest, which will be identified below, were found to fall into one of three categories. Internal factors are those which are to be calibrated to fixed values. Advanced factors are psychological and behavior traits, which should be recalibrated by a model expert for each scenario and should not usually be visible to the OR analyst. Model variables are those input factors which will be varied by the OR analyst in the specific scenario. Thus the calibration goal for the model variables is to identify valid variable ranges to be used by the OR analyst. Examples of factors identified in each of the three categories are depicted in Table 2. The variable input factors depicted in Table 2 determine how fast an individual's anger or level of arousal will rise in applicable situations.

Table 2: Examples of input factors divided into three categories

\begin{tabular}{|l|l|l|}
\hline Model Inputs & Description & Category \\
\hline $\begin{array}{l}\text { Threshold of insignific- } \\
\text { ance of a motive }\end{array}$ & $\begin{array}{l}\text { If a motivational power of a civilian (e.g. fear or anger) is below } \\
\text { this threshold, it will not motivate the civilian (and thus not trig- } \\
\text { ger any actions) even if it is stronger than any other motive. }\end{array}$ & Internal \\
\hline $\begin{array}{l}\text { Threshold for handing } \\
\text { over a package }\end{array}$ & $\begin{array}{l}\text { Determines the fear level a civilian must have before he/she hands } \\
\text { over a package to another civilian claiming it. }\end{array}$ & Internal \\
\hline $\begin{array}{l}\text { Persuasiveness of pacify- } \\
\text { ing actions }\end{array}$ & $\begin{array}{l}\text { Determines how effective reasonable talking ("pacifying") is in } \\
\text { making a civilian follow the current order (e.g. to go home). }\end{array}$ & Advanced \\
\hline Persuasiveness of threats & $\begin{array}{l}\text { Determines how effective threatening is in persuading a civilian } \\
\text { to follow the current order. }\end{array}$ & Advanced \\
\hline Persuasiveness of attacks & $\begin{array}{l}\text { Determines how effective attacks are in making a civilian do as } \\
\text { the attacker wishes. In most cases, a civilian will (try to) back off } \\
\text { into the tent area when attacked, for example. }\end{array}$ & Advanced \\
\hline Civilians' anger factor & $\begin{array}{l}\text { Determines the dynamics of the anger of an agent. A high anger } \\
\text { factor results in the anger rising faster, thus characterizing - to- } \\
\text { gether with other model inputs - a rather "choleric" civilian. }\end{array}$ & Variable \\
\hline Civilians' arousal factor & $\begin{array}{l}\text { Determines the dynamics of the arousal of an agent. A high } \\
\text { arousal factor results in the arousal rising faster, thus characteriz- } \\
\text { ing - together with other model inputs - a rather "hot-tempered" } \\
\text { civilian. }\end{array}$ & Variable \\
\hline
\end{tabular}

Although most of the factors in a simulation model of psychological aspects are hard to quantify, the table shows that the more "tangible" factors typically fall into the Variable category. Intuitively, these are factors that can be measured or at least assessed by an analyst. For example, to model an angry group of people hard to control the experienced analyst would set the anger factor to quite a high value (reasonable ranges will be given below).

On the other hand, the internal factors are those that the analyst will not want to deal with. They are usually very technical or psychological in nature (or both). To give an example, the threshold below which a motivational power of a civilian is considered insignificant is a factor that certainly has an impact on the model behavior, but is not meaningful to the analyst and should not be changed throughout different scenarios once calibrated to a value. 


\subsection{Run and Analyze Experiments to Develop Understanding and to Exclude Unrealistic Settings}

The goal of this phase was to develop a better functional understanding of the behavior of the scenario and to narrow down the ranges of all factors to reasonable intervals. For this purpose, several broad range experiments were done on a supercom-

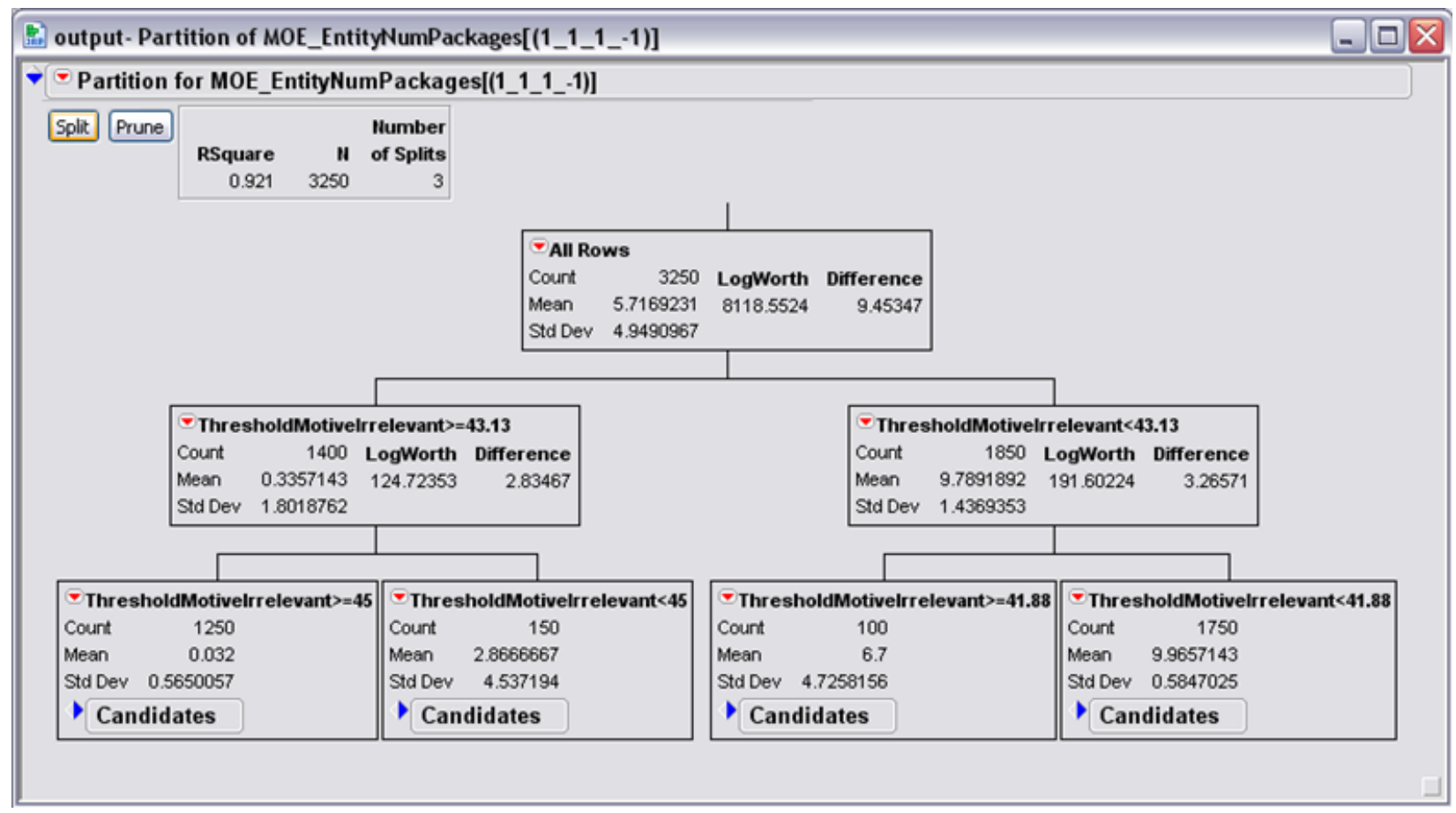

Figure 4: Regression tree analysis to help narrow down parameter ranges

puting cluster. One of the results was the finding of an appropriate level of the threshold below which a motive is considered irrelevant in the PAX behavior model (Threshold of insignificance of a motive). Regression tree analysis of the results of one experiment showed that this threshold was the predominant factor with respect to our MOE of Soldier's success in preventing stealing (measured by the number of packages that were stolen, see Figure 3). A visual study of unexpected model results via the model's animation and graphs of human emotions over time (as seen later in Figure 4) confirmed that the most realistic results are produced with this internal model factor fixed to a value of around 20, while still leaving enough influence for the advanced and variable factors - an important side condition when narrowing down parameter ranges.

Similar analyses indicated more factors to be fixed to specific values or bound to a certain parameter range. Those considerations formed the basis for the experiments conducted during the actual calibration phase, described in more detail in the following section.

\subsection{Calibration Phase}

The calibration phase encompasses the actual comparison of the experiments to the MOEs defined through subject matter expertise as well as adjustments to reduce ascertained discrepancies. For this purpose, a Nearly-Orthogonal Latin Hypercube (NOLH) design (Cioppa and Lucas 2007) with 15 factors was created and run for each vignette, based on the experiments in the previous phase and the parameters and ranges finally selected. Using a Latin Hypercube design allowed the simulation of multiple levels of each factor, without an extensive amount of simulation runs, which would be required by a general design such as a $2^{\mathrm{k}}$. Table 3 depicts a selection of those parameters with their respective value or parameter range, where the values and ranges of the internal and advanced factors had been pre-calibrated in the previous phase. 
Table 3: Parameter ranges in the NOLH design of calibration experiment Exp 01

\begin{tabular}{|l|l|r|}
\hline Model factor & Category & \multicolumn{1}{l|}{$\begin{array}{l}\text { Range in } \\
\text { NOLH DoE }\end{array}$} \\
\hline Threshold of insignificance of a motive & Internal & \multicolumn{1}{c|}{ fixed to 20 } \\
\hline Threshold for handing over a package & Internal & fixed to 70 \\
\hline Persuasiveness of attacks & Advanced & fixed to 100 \\
\hline Persuasiveness of pacifying & Advanced & {$[20 ; 50]$} \\
\hline Persuasiveness of threats & Advanced & {$[30 ; 60]$} \\
\hline Influence of a pacifying action on fear & Advanced & {$[-10 ; 20]$} \\
\hline Influence of a pacifying action against so. else on fear & Advanced & {$[-5 ; 20]$} \\
\hline Influence of a threatening action on fear & Advanced & {$[35 ; 55]$} \\
\hline Influence of a threatening action against so. else on fear & Advanced & {$[20 ; 50]$} \\
\hline Influence of a warning shot on fear & Advanced & {$[60 ; 90]$} \\
\hline Influence of a warning shot against so. else on fear & Advanced & {$[60 ; 85]$} \\
\hline Influence of an attack on fear & Advanced & {$[80 ; 90]$} \\
\hline Influence of an attack against so. else on fear & Advanced & {$[70 ; 95]$} \\
\hline Civilians' anger factor & Variable & {$[0.01 ; 0.3]$} \\
\hline Civilians' arousal factor & Variable & {$[1.0 ; 3.0]$} \\
\hline
\end{tabular}

The influence factors define the (additive) influence of an action on the respective model variable. For instance, the "influence of a warning shot on fear" defines the amount by which the fear level of the agent rises when a warning shot is fired against him or her.

The results from the first calibration experiment (Exp 01) are presented in Table 4. Exp 01 indicated a straight success for the high-escalation vignette since our calibration goal for each of the MOEs had been immediately achieved. The highescalation vignette was therefore declared calibrated after double-checking the DoE experiment by analyzing single simulation runs and verifying that the course of the simulation was plausible as well, in addition to the statistical achievement of the MOEs.

Table 4: Calibration results

\begin{tabular}{|l|l|l|l|}
\hline MOE & Goal & Exp 01 & Exp 02 \\
\hline Low-escalation vignette & $>90 \%$ & $3.01 \%$ & $43.26 \%$ \\
\hline Soldier's success in preventing stealing & $0 \%$ & $0 \%$ & $0 \%$ \\
\hline Use of weapons by CivA & $0 \%$ & $0 \%$ & $0 \%$ \\
\hline Use of weapons by soldier & $\geq 90 \%$ & $95.29 \%$ & - \\
\hline High-escalation vignette & $\geq 0 \%$ & - \\
\hline Soldier's success in preventing stealing
\end{tabular}

Exp 01 for the low-escalation vignette resulted in a surprise: the soldier performed extremely poorly in preventing $\operatorname{CivA}$ from stealing the packages of $\operatorname{CivB}$. More precisely, he was successful in only $3 \%$ of the cases! Analysis of the results indicated that this was due to the threshold for handing over package factor, which was subsequently fixed to a value of 1 for $\operatorname{Exp} 02$. While this re-calibration already resulted in an improvement to $43 \%$ (see Table 4 ), further investigation warranted changes on the implementation side to handle this particular type of situation. The team also studied the animation for several single runs (see Figure 4) and tracked behavior variables as a function of time to verify that the calibration results were not achieved arbitrarily or due to random effects. 


\section{Johnson, Lampe and Seichter}

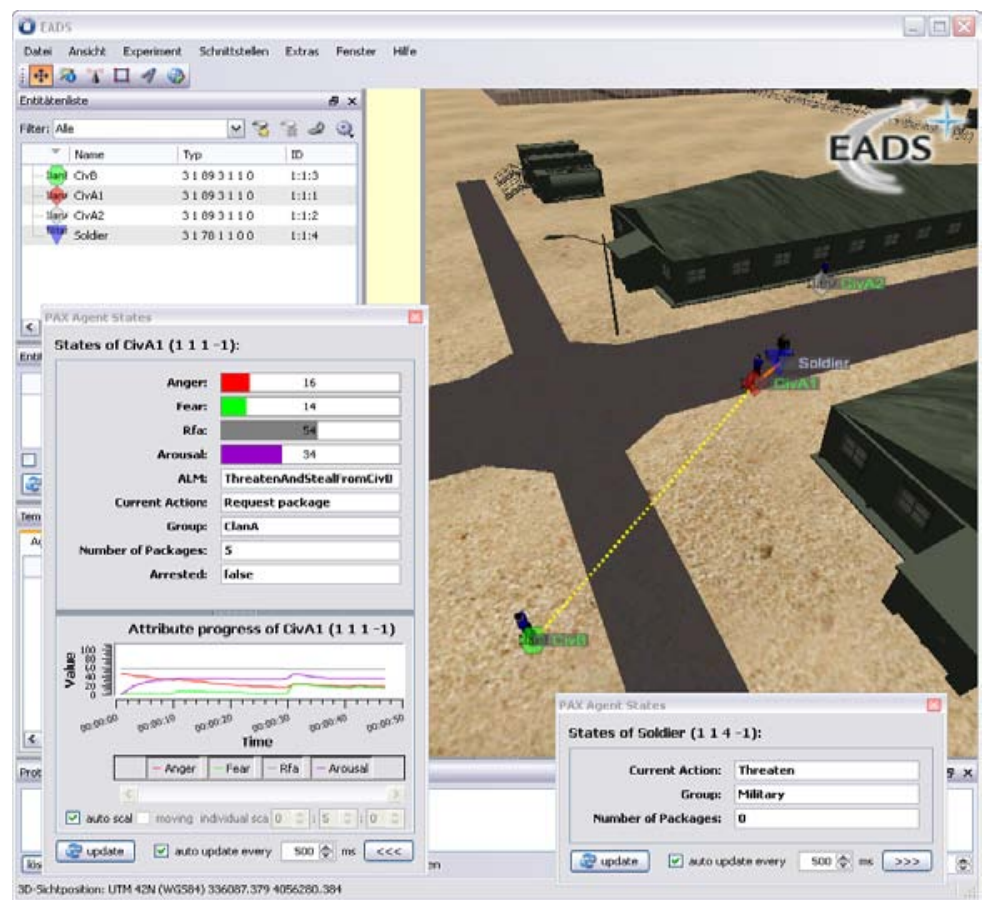

Figure 5: Detailed analysis of a single simulation run in the PAX GUI

\section{DISCUSSION}

Calibration is an iterative process that can be time consuming and difficult. Considering that a model as complex as PAX comprises research and development work of several years, calibrating the complex dynamics of the system is obviously challenging. However, the fact that a model with hundreds of parameters and highly complex dynamics is hard to calibrate and even harder to validate - does not mean that the task be forlorn hope.

This paper demonstrated a calibration methodology relying on plausibility considerations with the help of subject matter experts and making use of efficient experimental designs. We deem the presented approach particularly useful in conditions where real system data is hard to get or assess. This was illustrated in the calibration of an agent-based simulation model of a refugee camp situation. Quantitative data of the real system was not available, but subject matter expertise made up for this requirement up to a certain extent.

The most helpful lessons the researchers learned in this study were the following:

- Determination of different types of model input factors was essential in the calibration of the model. As an example, three main types of model factors were found in this study

- Internal model factors determine general human behavior traits and can be calibrated to fixed values

- Advanced model factors are used for scenario-specific model initialization and need to be re-calibrated when the scenario changes

- Model variables are those variables that will be changed by users of the simulation and the factors that are calibrated should be robust to these variables

- In the first step of the calibration, the factors should be varied widely to get a good understanding of the system.

Other methods that can complement the methodology demonstrated in this paper include the use of optimization algorithms, sensitivity analysis or scenario rotation techniques in which better statistical confidence is gained by having independent groups calibrate the same set of scenario vignettes. Given the aforementioned conditions, however, the approach as shown comprises the most appropriate techniques in a very time-efficient manner.

\section{ACKNOWLEDGEMENTS}

The PAX model was developed by EADS with assistance from the Operations Research Division of the Bundeswehr Transformation Center. PAX was initiated and funded by the German Bundeswehr. 


\section{REFERENCES}

Banks, J., J. S. Carson, B. L. Nelson, and D. M. Nicol. 2000. Discrete-Event System Simulation. 3rd ed. Upper Saddle River, New Jersey: Prentice-Hall, Inc.

Cioppa, T. M., and T. W. Lucas. 2007. Efficient Nearly Orthogonal and Space-Filling Latin Hypercubes. Technometrics, $49(1), 45-55$.

Mosler, H.-J., and G. Schwarz. 2005. Investigating Escalation Processes in Peace Support Operations: An Agent-based Model about Collective Aggression. In Proceedings of the 3rd Annual Conference of the European Social Simulation Association (ESSA 2005), Koblenz, Germany.

Sanchez, S. M., and T. W. Lucas. 2002. Exploring the World of Agent-Based Simulations: Simple Models, Complex Analyses. In Proceedings of the 2002 Winter Simulation Conference ed. E. Yücesan, C. H. Chen, J. L. Snowdon, J. M. Charnes, 116 - 126. Piscataway, New Jersey: Institute of Electrical and Electronics Engineers, Inc.

Urban, C. 2005. Das Referenzmodell PECS - Agentenbasierte Modellierung menschlichen Handelns, Entscheidens und Verhaltens. Dissertation, University of Passau, Germany.

\section{AUTHOR BIOGRAPHIES}

RACHEL T. JOHNSON is an Assistant Professor in the Operations Research Department at the Naval Postgraduate School. She received her PhD and MS in Industrial Engineering from Arizona State University and her BS in Industrial Engineering from Northwestern University. Her research interests are in the design and analysis of both physical and computer experiments and simulation methodology. She is a recipient of the Mary G. and Joseph Natrella Scholarship for excellence in statistics. She is a member of the American Society for Quality and the Institute for Operations Research and Management Sciences. Her email address is $<r t j o h n s o @ n p s$. edu> .

THORSTEN A. LAMPE is a Project Manager and System Designer in the System Design Centre Germany at EADS in Unterschleissheim. He holds a Diploma $(\sim \mathrm{BSc}+\mathrm{MSc})$ in Computer Science and his areas of expertise include Agent-Based Modeling and Simulation (ABMS) with special reference to modeling human behavior as well as experimentation methodologies (including Design of Experiments and optimization techniques) and service-oriented architectures (SOA). His email address is <thorsten. lampeeeads. com>.

STEPHAN SEICHTER graduated 2005 as Master of Science in Operations Research from the U.S. Naval Postgraduate School in Monterey, USA, and is currently working for Bundeswehr Transformation Center's Modeling \& Simulation $(\mathrm{M} \& \mathrm{~S})$ Department in the field of M\&S methodologies and fundamentals. The focus areas herein are simulation-based analysis methods and techniques like the Design of Experiments and the application of agent based modeling in support of applied Military Operations Research. His email address is <stephanseichter@bundeswehr.org >. 